Bull. Korean Math. Soc. 48 (2011), No. 3, pp. 445-454

DOI 10.4134/BKMS.2011.48.3.445

\title{
A DIFFERENTIAL EQUATION FOR MULTIPLE BESSEL POLYNOMIALS WITH RAISING AND LOWERING OPERATORS
}

\author{
Jin Ok Baek And Dong Won Lee
}

\begin{abstract}
In this paper, we first find a raising operator and a lowering operator for multiple Bessel polynomials and then give a differential equation having multiple Bessel polynomials as solutions. Thus the differential equations were found for all multiple orthogonal polynomials that are orthogonal with respect to the same type of classical weights introduced by Aptekarev et al.
\end{abstract}

\section{Introduction}

Let $\mathbb{N}_{0}:=\mathbb{N} \cup\{0\}, r \geq 2$ be a fixed integer, and $\vec{n}=\left(n_{1}, n_{2}, \ldots, n_{r}\right) \in \mathbb{N}_{0}^{r}$ be a multi-index with $|\vec{n}|=n_{1}+n_{2}+\cdots+n_{r}$. A sequence $\left\{P_{\vec{n}}(x)\right\}_{|\vec{n}|=0}^{\infty}$ of polynomials is called a multiple orthogonal polynomial system (multiple OPS) if

(i) $\operatorname{deg}\left(P_{\vec{n}}\right)=|\vec{n}|$;

(ii) there exist $r$ moment functionals $\sigma_{k}(k=1,2, \ldots, r)$ such that

$$
\left\langle\sigma_{k}, x^{j} P_{\vec{n}}(x)\right\rangle=\left\{\begin{array}{lll}
0 & \text { if } & j=0,1,2, \ldots, n_{k}-1, \\
\neq 0 & \text { if } & j=n_{k} .
\end{array}\right.
$$

In this case, $\sigma_{k}$ 's are called orthogonalizing moment functionals of multiple OPS $\left\{P_{\vec{n}}(x)\right\}_{|\vec{n}|=0}^{\infty}$.

The multiple OPS have been used in rational approximation in nineteenth century and recently applied in many fields such as number theory, rational approximation, dynamical system, and random matrix theory. Many properties of the multiple OPS are obtained as a generalization of ordinary orthogonal polynomials. See references $[2,3,15]$ and therein.

For the case of $r=2$, third order differential equations were found for multiple OPS's whose orthogonalizing moment functionals consist of the same type of classical moment functionals ([1]).

Received December 12, 2008; Revised May 6, 2010.

2010 Mathematics Subject Classification. 33C45, 33C47, 34L05, 42C05.

Key words and phrases. multiple orthogonal polynomials, Bessel polynomials, multiple Bessel polynomial, differential equation. 
More precisely, they established third order differential equations for the following multiple OPS's.

- The multiple Bessel polynomials $\left\{B_{\vec{n}}^{(\vec{a} ; b)}(x)\right\}$, where $a_{i}-a_{j} \notin \mathbb{Z}$ for $i \neq j$, whose orthogonalizing moment functional $\sigma_{k}$ satisfies

$$
\left(x^{2} \sigma_{k}\right)^{\prime}=\left[\left(a_{k}+2\right) x+b\right] \sigma_{k}, \quad k=1,2, \ldots, r .
$$

- The multiple Jacobi-Piñeiro polynomials $\left\{P_{\vec{n}}^{(\vec{\alpha} ; \alpha)}(x)\right\}$, where $\alpha, \alpha_{i}>$ -1 , and $\alpha_{i}-\alpha_{j} \notin \mathbb{Z}$ for $i \neq j$, whose orthogonalizing moment functional $\sigma_{k}$ satisfies

$$
\left(x(1-x) \sigma_{k}\right)^{\prime}=\left[\alpha_{k}+1-\left(\alpha_{k}+\alpha+2\right) x\right] \sigma_{k}, \quad k=1,2, \ldots, r .
$$

- The multiple Laguerre I polynomials $\left\{L_{\vec{n}}^{(\vec{\alpha} ; \beta)}(x)\right\}$, where $\alpha_{i}>-1, \beta>$ 0 , and $\alpha_{i}-\alpha_{j} \notin \mathbb{Z}$ for $i \neq j$, whose orthogonalizing moment functional $\sigma_{k}$ satisfies

$$
\left(x \sigma_{k}\right)^{\prime}=\left(\alpha_{k}+1-\beta x\right) \sigma_{k}, \quad k=1,2, \ldots, r .
$$

- The multiple Laguerre II polynomials $\left\{L_{\vec{n}}^{(\alpha ; \vec{\beta})}(x)\right\}$, where $\alpha>-1, \beta_{i}>$ 0 , and $\beta_{i} \neq \beta_{j}$ for $i \neq j$, whose orthogonalizing moment functional $\sigma_{k}$ satisfies

$$
\left(x \sigma_{k}\right)^{\prime}=\left(\alpha+1-\beta_{k} x\right) \sigma_{k}, \quad k=1,2, \ldots, r .
$$

- The multiple Hermite polynomials $\left\{H_{\vec{n}}^{(\delta ; \vec{b})}(x)\right\}$, where $\delta<0$, and $b_{i} \neq$ $b_{j}$ for $i \neq j$, whose orthogonalizing moment functional $\sigma_{k}$ satisfies

$$
\sigma_{k}^{\prime}=\left(\delta x+b_{k}\right) \sigma_{k}, \quad k=1,2, \ldots, r .
$$

See Table 1 and Table 2 in [1] for details.

They obtained third order differential equations using Rodrigues' formula and combinatorial identities. In [12], differential equations for multiple Hermite polynomials and multiple Laguerre polynomials were reconstructed using the generating functions. Recently, Coussement and Van Assche [5] found again the $(r+1)$ th order differential equations for multiple OPS's, except multiple Bessel polynomials, using lowering and raising operators.

In this paper, we first find a raising operator and a lowering operator for multiple Bessel polynomials and then give a $(r+1)$ th order differential equation having multiple Bessel polynomials as solutions. Thus the differential equations were found for all multiple OPS's that are orthogonal with respect to the same type of classical weights in [1].

\section{Main results}

Let's denote the $i$-th standard unit vector by $e_{i}=(0,0, \ldots, 1, \ldots, 0)$ and $e=(1,1, \ldots, 1)=e_{1}+e_{2}+\cdots+e_{r}$. Any linear functional $\sigma$ on the space of 
polynomials is called a moment functional and denote its action on a polynomial $\pi$ by

$$
\langle\sigma, \pi\rangle \text {. }
$$

For a moment functional $\sigma$ and a polynomial $\alpha$, we let $\sigma^{\prime}$ and $\alpha \sigma$ be the moment functionals defined by

$$
\left\langle\sigma^{\prime}, \pi\right\rangle=-\left\langle\sigma, \pi^{\prime}\right\rangle
$$

and

$$
\langle\alpha \sigma, \pi\rangle=\langle\sigma, \alpha \pi\rangle
$$

for every polynomial $\pi$.

Note that any orthogonalizing weight function $w$ defines a moment functional $\sigma$ by

$$
\left\langle\sigma, x^{k}\right\rangle=\int_{I} x^{k} w(x) d x,
$$

where $I$ is an interval on the real line $\mathbb{R}$. Due to Duran [6] for moment problem, for any moment functional $\sigma$ there exists a weight $w$ in Schwartz space that represent $\sigma$ by the integration of the form (2.1).

The orthogonality for Bessel polynomials was well known by $[8,9]$ in complex plane and later the real orthogonalizing weights were obtained in $[7,10,14]$, which gave an integral representation of the form (2.1) for the moment functional of Bessel polynomials. Moreover, the orthgonalizing moment functional $\sigma$ of Bessel polynomials satisfies a functional equation of the form

$$
\left(x^{2} \sigma\right)^{\prime}=[(a+2) x+b] \sigma
$$

and conversely any solution $\sigma$ of (2.2) orthogonalizes the Bessel polynomials when $a+2 \notin\{0,-1,-2, \ldots\}([4,11,16])$. Hence, the multiple Bessel polynomials $\left\{B_{\vec{n}}^{(\vec{a} ; b)}(x)\right\}$ is a sequence of polynomials such that

(i) $\operatorname{deg}\left(B_{\vec{n}}^{(\vec{a} ; b)}\right)=|\vec{n}|$;

(ii) for each $i=1,2, \ldots, r$,

$$
\left\langle\sigma_{i}, x^{j} B_{\vec{n}}^{(\vec{a} ; b)}(x)\right\rangle= \begin{cases}0 & \text { if } \quad j=0,1,2, \ldots, n_{i}-1, \\ \neq 0 & \text { if } \quad j=n_{i}\end{cases}
$$

where each $\sigma_{i}$ 's are the orthogonalizing moment functionals satisfying the equation of the form (2.2).

Here we assumed that $\vec{a}=\left(a_{1}, a_{2}, \ldots, a_{r}\right) \in \mathbb{R}^{r}, b \in \mathbb{R}, a_{i}+2 \notin\{0,-1,-2, \ldots\}$, and $a_{i}-a_{j} \notin \mathbb{Z}$ for $i \neq j$.

From here on, we always assume that $\sigma_{i}$ 's $(i=1,2, \ldots, r)$ are moment functionals satisfying the functional equation of the form (2.2). 
Theorem 2.1. For the multiple Bessel polynomials $\left\{B_{\vec{n}}^{(\vec{a} ; b)}(x)\right\}$, we have for $k=1,2, \ldots, r$,

$x^{2} \frac{d}{d x} B_{\vec{n}}^{\left(\vec{a}+e+e_{k} ; b\right)}(x)+\left[\left(a_{k}+2\right) x+b\right] B_{\vec{n}}^{\left(\vec{a}+e+e_{k} ; b\right)}(x)=\left(|\vec{n}|+a_{k}+2\right) B_{\vec{n}+e_{k}}^{(\vec{a} ; b)}(x)$.

Proof. Let $B_{\vec{n}}(x)=B_{\vec{n}}^{\left(\vec{a}+e+e_{k} ; b\right)}(x)$ and $\pi(x)=x^{2} B_{\vec{n}}^{\prime}(x)+\left[\left(a_{k}+2\right) x+b\right] B_{\vec{n}}(x)$. Since $B_{\vec{n}}$ is a monic polynomial of degree $|\vec{n}|, \pi$ is a polynomial of degree $|\vec{n}|+1$ with leading coefficient $|\vec{n}|+a_{k}+2$. Since $\left\{B_{\vec{n}}(x)\right\}_{|\vec{n}|=0}^{\infty}$ is orthogonal with respect to multiple moment functionals $\left(x \sigma_{1}, x \sigma_{2}, \ldots, x^{2} \sigma_{k}, \ldots, x \sigma_{r}\right)$, we have by integration by parts that for $i=k$,

$$
\begin{aligned}
\left\langle\sigma_{i}, x^{j} \pi(x)\right\rangle & =\left\langle\sigma_{k}, x^{j}\left[x^{2} B_{\vec{n}}^{\prime}(x)+\left(\left(a_{k}+2\right) x+b\right) B_{\vec{n}}(x)\right]\right\rangle \\
& =\left\langle\left[\left(a_{k}+2\right) x+b\right] x^{j} \sigma_{k}-\left(x^{j+2} \sigma_{k}\right)^{\prime}, B_{\vec{n}}(x)\right\rangle \\
& =-j\left\langle x^{j+1} \sigma_{k}, B_{\vec{n}}(x)\right\rangle \\
& =-j\left\langle x^{2} \sigma_{k}, x^{j-1} B_{\vec{n}}(x)\right\rangle=0, \quad j=0,1,2, \ldots, n_{i}
\end{aligned}
$$

and for $i \neq k$,

$$
\begin{aligned}
\left\langle\sigma_{i}, x^{j} \pi(x)\right\rangle & =\left\langle\sigma_{i}, x^{j}\left[x^{2} B_{\vec{n}}^{\prime}(x)+\left(\left(a_{k}+2\right) x+b\right) B_{\vec{n}}(x)\right]\right\rangle \\
& =\left\langle\left[\left(a_{k}+2\right) x+b\right] x^{j} \sigma_{i}-\left(x^{j+2} \sigma_{i}\right)^{\prime}, B_{\vec{n}}(x)\right\rangle \\
& =\left(a_{k}-a_{i}\right)\left\langle x^{j+1} \sigma_{i}, B_{\vec{n}}(x)\right\rangle-j\left\langle x^{j+1} \sigma_{i}, B_{\vec{n}}(x)\right\rangle \\
& =\left(a_{k}-a_{i}-j\right)\left\langle\sigma_{i}, x^{j+1} B_{\vec{n}}(x)\right\rangle \\
& =0, \quad j=0,1,2, \ldots, n_{i}-1 .
\end{aligned}
$$

Hence, $\left\langle\sigma_{i}, x^{j} \pi(x)\right\rangle=0$ for $j=0,1,2, \ldots, n_{k}$ when $i=k$, and $j=0,1,2, \ldots$, $n_{i}-1$ when $i \neq k$. By the uniqueness of the multiple orthogonal polynomials, we can write

$$
\pi(x)=c_{n} B_{\vec{n}+e_{k}}^{(\vec{a} ; b)}(x),
$$

where $c_{n}$ is a constant. Since $B_{\vec{n}+e_{k}}^{(\vec{a} ; b)}(x)$ is monic, by comparing the leading coefficients, we obtain the result.

If we define an operator $L_{a_{k}}$ by

$$
L_{a_{k}}[y](x)=x^{2} \frac{d y}{d x}+\left[\left(a_{k}+2\right) x+b\right] y,
$$

then Theorem 2.1 gives a raising operator

$$
L_{a_{k}}\left[B_{\vec{n}}^{\left(\vec{a}+e+e_{k} ; b\right)}\right](x)=\left(|\vec{n}|+a_{k}+2\right) B_{\vec{n}+e_{k}}^{(\vec{a} ; b)}(x) .
$$

In order to obtain a lowering operator, we need a lemma whose proof is quite simple $([13])$.

Lemma 2.2. Let $C=\left[c_{i j}\right]_{i, j=1}^{r}$ be a matrix. If

$$
c_{i p}-c_{i q}=\epsilon_{p q} c_{i p} c_{i q} \quad \text { and } \quad c_{p i}-c_{q i}=\delta_{p q} c_{p i} c_{q i}, \quad i, p, q=1,2, \ldots, r
$$


then the determinant $|C|$ of $C$ is

$$
|C|=\left(\prod_{i=1}^{r} \prod_{j=1}^{r} c_{i j}\right)\left(\prod_{q=1}^{r-1} \prod_{p=q+1}^{r} \epsilon_{p q} \delta_{p q}\right) .
$$

Proof. It can be inductively proved by row and column operations as following:

$$
\begin{aligned}
& \left|\begin{array}{cccc}
c_{11} & c_{12} & \cdots & c_{1 r} \\
c_{21} & c_{22} & \cdots & c_{2 r} \\
\vdots & \vdots & \ddots & \vdots \\
c_{r 1} & c_{r 2} & \cdots & c_{r r}
\end{array}\right|=\left|\begin{array}{cccc}
c_{11} & c_{12}-c_{11} & \cdots & c_{1 r}-c_{11} \\
c_{21} & c_{22}-c_{21} & \cdots & c_{2 r}-c_{21} \\
\vdots & \vdots & \ddots & \vdots \\
c_{r 1} & c_{r 2}-c_{r 1} & \cdots & c_{r r}-c_{r 1}
\end{array}\right| \\
& =\left|\begin{array}{cccc}
c_{11} & \epsilon_{21} c_{11} c_{12} & \cdots & \epsilon_{r 1} c_{11} c_{1 r} \\
c_{21} & \epsilon_{21} c_{21} c_{22} & \cdots & \epsilon_{r 1} c_{21} c_{2 r} \\
\vdots & \vdots & \ddots & \vdots \\
c_{r 1} & \epsilon_{21} c_{r 1} c_{r 2} & \cdots & \epsilon_{r 1} c_{r 1} c_{r r}
\end{array}\right| \\
& =\prod_{i=1}^{r} c_{i 1} \prod_{i=2}^{r} \epsilon_{i 1}\left|\begin{array}{cccc}
1 & c_{12} & \cdots & c_{1 r} \\
1 & c_{22} & \cdots & c_{2 r} \\
\vdots & \vdots & \ddots & \vdots \\
1 & c_{r 2} & \cdots & c_{r r}
\end{array}\right| \\
& =\prod_{i=1}^{r} c_{i 1} \prod_{i=2}^{r} \epsilon_{i 1}\left|\begin{array}{cccc}
1 & c_{12} & \cdots & c_{1 r} \\
0 & \delta_{21} c_{12} c_{22} & \cdots & \delta_{21} c_{1 r} c_{2 r} \\
\vdots & \vdots & \ddots & \vdots \\
0 & \delta_{r 1} c_{12} c_{r 2} & \cdots & \delta_{r 1} c_{1 r} c_{r r}
\end{array}\right| \\
& =\left(\prod_{i=1}^{r} c_{i 1}\right)\left(\prod_{j=2}^{r} c_{1 j}\right)\left(\prod_{i=2}^{r} \epsilon_{i 1} \delta_{i 1}\right)\left|\begin{array}{cccc}
c_{22} & c_{23} & \cdots & c_{2 r} \\
c_{32} & c_{33} & \cdots & c_{3 r} \\
\vdots & \vdots & \ddots & \vdots \\
c_{r 2} & c_{r 3} & \cdots & c_{r r}
\end{array}\right|
\end{aligned}
$$

Theorem 2.3. For the multiple Bessel polynomials $\left\{B_{\vec{n}}^{(\vec{a} ; b)}(x)\right\}$, we have

$$
\frac{d}{d x}\left(B_{\vec{n}}^{(\vec{a} ; b)}(x)\right)=\sum_{k=1}^{r} d_{k} B_{\vec{n}-e_{k}}^{\left(\vec{a}+e+e_{k} ; b\right)}(x),
$$

where

$$
\begin{aligned}
d_{k}= & \frac{\prod_{p=1}^{r}\left(n_{p}+a_{p}-a_{k}\right)}{\left(|\vec{n}|+a_{k}+1\right) \prod_{q=1, q \neq k}^{r-1}\left(a_{k}-a_{q}\right) \prod_{p=k+1}^{r}\left(a_{p}-a_{k}\right)} \\
& \times \sum_{j=1}^{r} \frac{(-1)^{k+j}\left(n_{j}+a_{j}+1\right) \prod_{q=1}^{r}\left(n_{j}+a_{j}-a_{q}\right)}{\left(n_{j}+a_{j}-a_{k}\right) \prod_{q=1, q \neq j}^{r-1}\left(n_{q}-n_{j}+a_{q}-a_{j}\right) \prod_{p=j+1}^{r}\left(n_{j}-n_{p}+a_{j}-a_{p}\right)} .
\end{aligned}
$$


In particular, if $r=2$,

$$
\begin{aligned}
\frac{d}{d x}\left(B_{n_{1}, n_{2}}^{\left(a_{1}, a_{2} ; b\right)}(x)\right)= & \frac{n_{1}\left(n_{2}+a_{2}-a_{1}\right)}{a_{2}-a_{1}} B_{n_{1}-1, n_{2}}^{\left(a_{1}+2, a_{2}+1 ; b\right)}(x) \\
& -\frac{n_{2}\left(n_{1}+a_{1}-a_{2}\right)}{a_{2}-a_{1}} B_{n_{1}, n_{2}-1}^{\left(a_{1}+1, a_{2}+2 ; b\right)}(x) .
\end{aligned}
$$

Proof. Let $V$ be the space of polynomial $\pi$ such that $\operatorname{deg}(\pi)=|\vec{n}|-1$ and

$$
\left\langle\sigma_{i}, x^{j+2} \pi(x)\right\rangle=0, \quad j=0,1,2, \ldots, n_{i}-2, \quad i=1,2, \ldots, r .
$$

By the orthogonality of $\left\{B_{\vec{n}}^{\left(\vec{a}+e+e_{k} ; b\right)}(x)\right\}_{|\vec{n}|=0}^{\infty}$ with respect to $\left(x \sigma_{1}, x \sigma_{2}, \ldots\right.$, $\left.x^{2} \sigma_{k}, \ldots, x \sigma_{r}\right)$, it can be easily seen that

$$
B_{\vec{n}-e_{k}}^{\left(\vec{a}+e+e_{k} ; b\right)} \in V, \quad k=1,2, \ldots, r .
$$

By the linearly independency of $\left\{B_{\vec{n}-e_{k}}^{\left(\vec{a}+e+e_{k} ; b\right)}\right\}_{k=1}^{r}$, any polynomial $\pi$ in $V$ can be written by a linear combination of them. On the other hand, since $\left(x^{j+2} \sigma_{i}\right)^{\prime}=\left[\left(j+a_{i}+2\right) x+b\right] x^{j} \sigma_{i}$, we have

$$
\begin{aligned}
\left\langle\sigma_{i}, x^{j+2} \frac{d}{d x}\left(B_{\vec{n}}^{(\vec{a} ; b)}(x)\right)\right\rangle & =-\left\langle\sigma_{i},\left[\left(j+a_{i}+2\right) x+b\right] x^{j} B_{\vec{n}}^{(\vec{a} ; b)}(x)\right\rangle \\
& =0, \quad j=0,1,2, \ldots, n_{i}-2,
\end{aligned}
$$

which implies $\frac{d}{d x}\left(B_{\vec{n}}^{(\vec{a} ; b)}(x)\right) \in V$. Hence, we have

$$
\frac{d}{d x}\left(B_{\vec{n}}^{(\vec{a} ; b)}(x)\right)=\sum_{k=1}^{r} d_{k} B_{\vec{n}-e_{k}}^{\left(\vec{a}+e+e_{k} ; b\right)}(x),
$$

where $d_{k}$ 's are constants. In order to obtain $d_{k}$, multiplying $(2.5)$ by $x^{n_{i}-1}$ and then applying $x^{2} \sigma_{i}$ on both sides, we have

$$
\left\langle\sigma_{i}, x^{n_{i}+1} \frac{d}{d x}\left(B_{\vec{n}}^{(\vec{a} ; b)}(x)\right)\right\rangle=\sum_{k=1}^{r} d_{k}\left\langle\sigma_{i}, x^{n_{i}+1} B_{\vec{n}-e_{k}}^{\left(\vec{a}+e+e_{k} ; b\right)}(x)\right\rangle .
$$

On the other hand, by the raising operator, by the orthogonality, and by the moment equation, we obtain

$$
\begin{aligned}
& \left\langle\sigma_{i}, x^{n_{i}+1} B_{\vec{n}-e_{k}}^{\left(\vec{a}+e+e_{k} ; b\right)}(x)\right\rangle \\
= & \frac{1}{|\vec{n}|+a_{i}+1}\left\langle\sigma_{i}, x^{n_{i}+1} L_{a_{i}+1}\left[B_{\vec{n}-e_{k}-e_{i}}^{\left(\vec{a}+2 e+e_{k}+e_{i} ; b\right)}\right](x)\right\rangle \\
= & \frac{1}{\left(|\vec{n}|+a_{i}+1\right)}\left\{\left\langle\sigma_{i},\left[\left(a_{i}+3\right) x+b\right] x^{n_{i}+1} B_{\vec{n}-e_{k}-e_{i}}^{\left(\vec{a}+2 e+e_{i}+e_{i} ; b\right)}(x)\right\rangle\right. \\
& \left.\quad-\left\langle\left(x^{n_{i}+3} \sigma_{i}\right)^{\prime}, B_{\vec{n}-e_{k}-e_{i}}^{\left(\vec{a}+2 e+e_{k}+e_{i} ; b\right)}(x)\right\rangle\right\} \\
= & -\frac{n_{i}}{\left(|\vec{n}|+a_{i}+1\right)}\left\langle\sigma_{i}, x^{n_{i}+2} B_{\vec{n}-e_{k}-e_{i}}^{\left(\vec{a}+2 e+e_{i}+e_{i} ; b\right)}(x)\right\rangle \\
= & -\frac{n_{i}}{\left(|\vec{n}|+a_{i}+1\right)\left(|\vec{n}|+a_{1}+1\right)}\left\langle\sigma_{i}, x^{n_{i}+2} L_{a_{1}+2}\left[B_{\vec{n}-e_{1}-e_{k}-e_{i}}^{\left(\vec{a}+3 e+e_{i}+e_{k}+b\right)}\right](x)\right\rangle
\end{aligned}
$$




$$
\begin{gathered}
=-\frac{n_{i}}{\left(|\vec{n}|+a_{i}+1\right)\left(|\vec{n}|+a_{1}+1\right)}\left\{\left\langle\sigma_{i},\left[\left(a_{1}+4\right) x+b\right] x^{n_{i}+2} B_{\vec{n}-e_{1}-e_{k}-e_{i}}^{\left(\vec{a}+3 e+e_{i} ; b\right)}(x)\right\rangle\right. \\
\left.-\left\langle\left(x^{n_{i}+4} \sigma_{i}\right)^{\prime}, B_{\vec{n}-e_{1}-e_{k}-e_{i}}^{\left(\vec{a}+3 e+e_{i}+e_{i} ; b\right)}(x)\right\rangle\right\} \\
=-\frac{n_{i}\left(a_{1}-a_{i}-n_{i}\right)}{\left(|\vec{n}|+a_{i}+1\right)\left(|\vec{n}|+a_{1}+1\right)}\left\langle\sigma_{i}, x^{n_{i}+3} B_{\vec{n}-e_{1}-e_{k}-e_{i}}^{\left(\vec{a}+3 e+e_{1}+e_{i}+e_{i} ;\right)}(x)\right\rangle .
\end{gathered}
$$

Continuing the same process for the index from 1 to $r$, we obtain

$$
\left\langle\sigma_{i}, x^{n_{i}+1} B_{\vec{n}-e_{k}}^{\left(\vec{a}+e+e_{k} ; b\right)}(x)\right\rangle=\left(\prod_{p=1}^{r} \frac{a_{p}-a_{i}-n_{i}}{|\vec{n}|+a_{p}+1}\right) \frac{|\vec{n}|+a_{k}+1}{a_{k}-a_{i}-n_{i}} I_{i},
$$

where

$$
I_{i}=\left\langle\sigma_{i}, x^{n_{i}+r} B_{\vec{n}-e}^{(\vec{a}+(r+1) e ; b)}(x)\right\rangle .
$$

By the same method as above, we can easily see that

$$
\begin{aligned}
\left\langle\sigma_{i}, x^{n_{i}+1} \frac{d}{d x}\left(B_{\vec{n}}^{(\vec{a} ; b)}(x)\right)\right\rangle= & -\left\langle\left(x^{n_{i}+1} \sigma_{i}\right)^{\prime}, B_{\vec{n}}^{(\vec{a} ; b)}(x)\right\rangle \\
= & -\left\langle\left(x^{2} \sigma_{i}\right)^{\prime}, x^{n_{i}-1} B_{\vec{n}}^{(\vec{a} ; b)}(x)\right\rangle \\
& -\left(n_{i}-1\right)\left\langle\sigma_{i}, x^{n_{i}} B_{\vec{n}}^{(\vec{a} ; b)}(x)\right\rangle \\
= & -\left\langle\sigma_{i},\left[\left(n_{i}+a_{i}+1\right) x+b\right] x^{n_{i}-1} B_{\vec{n}}^{(\vec{a} ; b)}(x)\right\rangle \\
= & -\left(n_{i}+a_{i}+1\right)\left\langle\sigma_{i}, x^{n_{i}} B_{\vec{n}}^{(\vec{a} ; b)}(x)\right\rangle \\
= & -\left(n_{i}+a_{i}+1\right)\left(\prod_{p=1}^{r} \frac{a_{p}-a_{i}-n_{i}}{|\vec{n}|+a_{p}+1}\right) I_{i} .
\end{aligned}
$$

Since $I_{i} \neq 0$ by the orthogonality, substituting (2.7) and (2.8) into (2.6), we have

$$
\left(n_{i}+a_{i}+1\right)=\sum_{k=1}^{r} d_{k} \frac{\left(|\vec{n}|+a_{k}+1\right)}{\left(n_{i}+a_{i}-a_{k}\right)} \quad(i=1,2, \ldots, r)
$$

which is equivalent to

$$
A d=h,
$$

where $A:=\left[a_{i k}\right]_{i, k=1}^{r}$ is the $r \times r$ matrix with

$$
a_{i k}=\frac{|\vec{n}|+a_{k}+1}{n_{i}+a_{i}-a_{k}}
$$

and

$$
d=\left(\begin{array}{c}
d_{1} \\
d_{2} \\
\vdots \\
d_{r}
\end{array}\right), \quad h=\left(\begin{array}{c}
n_{1}+a_{1}+1 \\
n_{2}+a_{2}+1 \\
\vdots \\
n_{r}+a_{r}+1
\end{array}\right)
$$


By Lemma 2.2, we have

$$
|A|=\frac{\left\{\prod_{i=1}^{r}\left(|\vec{n}|+a_{i}+1\right)\right\}\left\{\prod_{q=1}^{r-1} \prod_{p=q+1}^{r}\left(a_{p}-a_{q}\right)\left(n_{q}-n_{p}+a_{q}-a_{p}\right)\right\}}{\prod_{q=1}^{r} \prod_{p=1}^{r}\left(n_{p}+a_{p}-a_{q}\right)} .
$$

On the other hand, the determinant of the matrix replaced $k$-th column by $h$, we obtain

$$
\left|\begin{array}{ccccc}
\frac{|\vec{n}|+a_{1}+1}{n_{1}} & \cdots & \left(n_{1}+a_{1}+1\right) & \cdots & \frac{|\vec{n}|+a_{r}+1}{n_{1}+a_{1}-a_{r}} \\
\frac{|\vec{n}|+a_{1}+1}{n_{2}+a_{2}-a_{1}} & \cdots & \left(n_{2}+a_{2}+1\right) & \cdots & \frac{|\vec{n}|+a_{r}+1}{n_{2}+a_{2}-a_{r}} \\
\vdots & \ddots & \vdots & \cdots & \vdots \\
\frac{|\vec{n}|+a_{1}+1}{n_{r}+a_{r}-a_{1}} & \cdots & \left(n_{r}+a_{r}+1\right) & \cdots & \frac{|\vec{n}|+a_{r}+1}{n_{r}}
\end{array}\right|=\sum_{j=1}^{r}(-1)^{k+j}\left|C_{j k}\right|
$$

where $C_{j k}$ is the determinant of $(j, k)$-minor of $A$. More precisely, we obtain by Lemma 2.2 ,

$$
\begin{aligned}
& \left|C_{j k}\right|=\left(n_{j}+a_{j}+1\right) \prod_{i=1, i \neq k}^{r}\left(|\vec{n}|+a_{i}+1\right) \\
& \times\left|\begin{array}{cccccc}
\frac{1}{n_{1}} & \cdots & \frac{1}{n_{1}+a_{1}-a_{k-1}} & \frac{1}{n_{1}+a_{1}-a_{k+1}} & \cdots & \frac{1}{n_{1}+a_{1}-a_{r}} \\
\frac{1}{n_{2}+a_{2}-a_{1}} & \cdots & \frac{1}{n_{2}+a_{2}-a_{k-1}} & \frac{1}{n_{2}+a_{2}-a_{k+1}} & \cdots & \frac{1}{n_{2}+a_{2}-a_{r}} \\
\vdots & \ddots & \vdots & \vdots & \ddots & \vdots \\
\frac{1}{n_{j-1}+a_{j-1}-a_{1}} & \cdots & \frac{1}{n_{j-1}+a_{j-1}-a_{k-1}} & \frac{1}{n_{j-1}+a_{j-1}-a_{k+1}} & \cdots & \frac{1}{n_{j-1}+a_{j-1}-a_{r}} \\
\frac{1}{n_{j+1}+a_{j+1}-a_{1}} & \cdots & \frac{1}{n_{j+1}+a_{j+1}-a_{k-1}} & \frac{1}{n_{j+1}+a_{j+1}-a_{k+1}} & \cdots & \frac{1}{n_{j+1}+a_{j+1}-a_{r}} \\
\vdots & \ddots & \vdots & \vdots & \ddots & \vdots \\
\frac{1}{n_{r}+a_{r}-a_{1}} & \cdots & \frac{1}{n_{r}+a_{r}-a_{k-1}} & \frac{1}{n_{r}+a_{r}-a_{k+1}} & \cdots & \frac{1}{n_{r}}
\end{array}\right| \\
& =\left(n_{j}+a_{j}+1\right) \prod_{i=1, i \neq k}^{r}\left(|\vec{n}|+a_{i}+1\right) \\
& \times \frac{\left\{\prod_{q=1, q \neq k}^{r-1} \prod_{p=q+1, p \neq k}^{r}\left(a_{p}-a_{q}\right)\right\}\left\{\prod_{q=1, q \neq j}^{r-1} \prod_{p=q+1, p \neq j}^{r}\left(n_{q}-n_{p}+a_{q}-a_{p}\right)\right\}}{\prod_{q=1, q \neq k}^{r} \prod_{p=1, p \neq j}^{r}\left(n_{p}+a_{p}-a_{q}\right)} .
\end{aligned}
$$

By Cramer's rule, we obtain $d_{k}=\frac{1}{|A|} \sum_{j=1}^{r}(-1)^{k+j}\left|C_{j k}\right|$, which is the result.

Combining the result of Theorem 2.1 and Theorem 2.3, we find a $(r+1)$ th order differential equation of multiple Bessel polynomials. 
Theorem 2.4. The multiple Bessel polynomial $\left\{B_{\vec{n}}^{(\vec{a} ; b)}(x)\right\}_{|\vec{n}|=0}^{\infty}$ satisfies a $(r+$ 1) th order differential equation

$$
\begin{aligned}
& L_{a_{1}-r+1} L_{a_{2}-r+2} \cdots L_{a_{r}}\left[y^{\prime}\right](x) \\
= & \sum_{k=1}^{r} d_{k}\left(|\vec{n}|+a_{k}+1\right) L_{a_{1}-r+1} \cdots L_{a_{k-1}-r+k-1} L_{a_{k+1}-r+k} \cdots L_{a_{r}-1}[y](x),
\end{aligned}
$$

where $y=B_{\vec{n}}^{(\vec{a} ; b)}(x)$ and $d_{k}$ 's are the constants in (2.4).

Proof. Since

$$
\begin{aligned}
L_{a_{j}} L_{a_{i}+1}[y]= & x^{4} y^{\prime \prime}+\left[\left(a_{i}+a_{j}+7\right) x^{3}+2 b x^{2}\right] y^{\prime} \\
& +\left[\left(a_{i} a_{j}+3 a_{i}+3 a_{j}+9\right) x^{2}+b\left(a_{i}+a_{j}+5\right) x+b^{2}\right] y \\
= & L_{a_{i}} L_{a_{j}+1}[y]
\end{aligned}
$$

we have

$$
\begin{aligned}
& L_{a_{1}-r+1} L_{a_{2}-r+2} \cdots L_{a_{i}-r+i} \cdots L_{a_{r}} \\
= & L_{a_{1}-r+1} L_{a_{2}-r+2} \cdots L_{a_{i-1}-r+i-1} L_{a_{i+1}-r+i} \cdots L_{a_{r}-1} L_{a_{i}} .
\end{aligned}
$$

Applying $L_{a_{1}-r+1} L_{a_{2}-r+2} \cdots L_{a_{r}}$ on (2.3) and Theorem 2.1, the conclusion follows.

When $r=2$, the differential equation (2.9) becomes

$$
L_{a_{1}-1} L_{a_{2}}\left[y^{\prime}\right]=d_{1}\left(|\vec{n}|+a_{1}+1\right) L_{a_{2}-1}[y]+d_{2}\left(|\vec{n}|+a_{2}+1\right) L_{a_{1}-1}[y]
$$

so that $B_{n_{1}, n_{2}}^{\left(a_{1}, a_{2} ; b\right)}(x)$ satisfies

$$
\begin{aligned}
x^{4} y^{\prime \prime \prime} & +\left[\left(a_{1}+a_{2}+5\right) x^{3}-2 b x^{2}\right] y^{\prime \prime}+\left[\left\{\left(a_{1}-n_{2}+2\right)\left(a_{2}-n_{1}+2\right)\right.\right. \\
& \left.\left.-\left(n_{1}+n_{2}\right)\left(n_{1}+n_{2}-1\right)\right\} x^{2}-\left(a_{1}+a_{2}+3\right) b x+b^{2}\right] y^{\prime} \\
& -\left[\left(n_{1}+n_{2}\right)\left(n_{1}+a_{1}+1\right)\left(n_{2}+a_{2}+1\right) x\right. \\
& \left.-b\left\{n_{1} n_{2}+n_{1}\left(n_{1}+a_{1}+1\right)+n_{2}\left(n_{2}+a_{2}+1\right)\right\}\right] y=0,
\end{aligned}
$$

which was already obtained in [1].

Acknowledgement. This work was supported by Korea Research Foundation Grant funded by the Korean Government (KRF-2008-313-C00027).

\section{References}

[1] A. I. Aptekarev, A. Branquinho, and W. Van Assche, Multiple orthogonal polynomials for classical weights, Trans. Amer. Math. Soc. 355 (2003), no. 10, 3887-3914.

[2] B. Beckermann, J. Coussement, and W. Van Assche, Multiple Wilson and Jacobi-Piñeiro polynomials, J. Approx. Theory 132 (2005), no. 2, 155-181.

[3] M. G. de Bruin, Simultaneous Padé approximation and orthogonality, Orthogonal polynomials and applications (Bar-le-Duc, 1984), 74-83, Lecture Notes in Math., 1171, Springer, Berlin, 1985.

[4] T. S. Chihara, An Introduction to Orthogonal Polynomials, Gordon and Breach, New York, 1978. 
[5] J. Coussement and W. Van Assche, Differential equations for multiple orthogonal polynomials with respect to classical weights: raising and lowering operators, J. Phys. A 39 (2006), no. 13, 3311-3318.

[6] A. J. Duran, The Stieltjes moment problem for rapidly decreasing functions, Proc. Amer. Math. Soc. 107 (1989), no. 3, 731-741.

[7] W. D. Evans, W. N. Everitt, K. H. Kwon, and L. L. Littlejohn, Real orthogonalizing weights for Bessel polynomials, J. Comput. Appl. Math. 49 (1993), no. 1-3, 51-57.

[8] A. M. Krall, Orthogonal polynomials through moment generating functionals, SIAM J. Math. Anal. 9 (1978), no. 4, 600-603.

[9] H. L. Krall and O. Frink, A new class of orthogonal polynomials: The Bessel polynomials, Trans. Amer. Math. Soc. 65 (1949), 100-115.

[10] K. H. Kwon, S. S. Kim, and S. S. Han, Orthogonalizing weights of Tchebychev sets of polynomials, Bull. London Math. Soc. 24 (1992), no. 4, 361-367.

[11] K. H. Kwon, D. W. Lee, and L. L. Littlejohn, Differential equations having orthogonal polynomial solutions, J. Comput. Appl. Math. 80 (1997), no. 1, 1-16.

[12] D. W. Lee, Properties of multiple Hermite and multiple Laguerre polynomials by the generating function, Integral Transforms Spec. Funct. 18 (2007), no. 11-12, 855-869.

[13] _ Difference equations for discrete classical multiple orthogonal polynomials, J. Approx. Theory 150 (2008), no. 2, 132-152.

[14] R. D. Morton and A. M. Krall, Distributional weight functions for orthogonal polynomials, SIAM J. Math. Anal. 9 (1978), no. 4, 604-626.

[15] E. M. Nikishin and V. N. Sorokin, Rational Approximations and Orthogonality, Translations of Mathematical Monographs, Amer. Math. Soc., Providence, RI 92, 1991.

[16] G. Szegö, Orthogonal Polynomials, 4th ed., Amer. Math. Soc., Colloq. Publ. 213, Providence, RI, 1975.

JIN OK BAEK

Department of Mathematics

KYUNGPOOK NATIONAL UNIVERSITY

TAEGU 702-701, KOREA

E-mail address: jbaek0125@knu.ac.kr

DONG WON LEE

Department of Mathematics

Teachers College

KYUnGPOOK NATIONAL University

TAEGU 702-701, KoreA

E-mail address: dongwon@knu.ac.kr 